\title{
Quantification of Viable Aerobic Bacteria in Oral Squamous Cell Carcinoma Tissue - A Microbiological Approach
}

\author{
${ }^{1}$ Dr. Supratim Ghosh, ${ }^{2}$ Dr. Minal Chaudhary, ${ }^{3}$ Dr. Swatil Patil, \\ ${ }^{1,2,3}$ B.D.S., M.D.S., \\ ${ }^{I}$ Clinical Tutor, Dept. Of Oral Pathology \& Microbiology, North Bengal Dental College \& Hospital \\ Sushrutanagar, Darjeeling, Pin-734012, West Bengal, India \\ ${ }^{2}$ Professor \& Head, Department of Oral Pathology \& Microbiology, Sharad Pawar Dental College \& Hospital, \\ Sawangi (M),Wardha- 442002, Maharashtra, India \\ ${ }^{3}$ Professor, Department of Oral Pathology \& Microbiology, Sharad Pawar Dental College \& Hospital, \\ Sawangi(M),Wardha- 442002, Maharashtra, India
}

\begin{abstract}
Despite increasing interest in the possible relationships between bacteria and the different stages of cancer development, the association of bacteria with cancer of the oral cavity has yet to be adequately examined. With that in mind, the primary objective of our study was to determine microbiologically the presence of viable aerobic bacteria in the lesional tissue of diagnosed cases of oral squamous cell carcinoma \& in apparently normal tissue atleast $5 \mathrm{~cm}$. away from the cancerous lesion and to determine presence of viable aerobic bacteria in normal tissue surgically removed from patients undergoing surgery for non-neoplastic \& non-inflammed lesions and then to compare among them. Cancerous lesions were removed surgically, and resected OSCC specimens were harvested under aseptic conditions. The specimen was placed on a sterile surface \& with a new blade for each cut a $1 \mathrm{~cm}^{3}$ specimen was removed without compromising the pathological margins. The specimen was further divided into upper level, middle level and lower level of the lesion. Whenever possible, a control specimen consisting of non-tumourous tissues harvested at least $5 \mathrm{~cm}$ away from the primary lesion was also obtained. All subsequent handling of the specimens was carried out using aseptic technique on surfaces cleaned with phenolic disinfectant. Tissue specimens were placed in betadine for 3 mins to disinfect the surfaces of each. Subsequently, tissues are placed in multiple labeled bottles of PBS (phosphatebuffered saline) to encourage the removal of any bacteria on the tissue surface and stored in deep freezer. Successful surface decontamination of the specimens indicates that the bacteria detected were from within the tissue. Bacteria have been cultured from within oral squamous cell carcinoma tissue. The significance of these bacteria within the tumor warrants further study.
\end{abstract}

Keywords: Bacteria, Oral squamous cell carcinoma (OSCC), serial dilution

\section{Introduction}

The association of bacteria with oral tumors is of increasing interest. In a study of intraoral carcinomas, a difference in the microflora associated with the surface of tumors in comparison to control sites was demonstrated. More recently it has also been reported that patients with OSCC tend to possess significantly raised concentrations of certain bacteria in their saliva - potential application as a diagnostic tool to predict oral cancer. Furthermore, sufficient attention must be given to the elimination from any tissues tested of the microbes that occur naturally on the surfaces of the tumors. In addition, salivary contamination of the sample must be prevented during subsequent handling. ${ }^{5}$

\section{Material \& Methods:}

The present study was carried in the Department of Oral Pathology and Microbiology, Sharad Pawar Dental College \& Hospital, DMIMS, Wardha (MS), India. The study consisted of a total test group of 30 patients diagnosed with OSCC.

2.1 Study group - 30 OSCC patients were included in the study group of which 25 were males and 5 were females, between the age group of 20 to 70 years.

2.2 Test group -

2.2.1 Group A: 30 individuals with clinically and histopathological diagnosed oral squamous cell carcinoma.

2.3 Control group -

2.3.1 Group B: Apparently normal tissue surgically removed from 10 individuals of group A from atleast $5 \mathrm{~cm}$ away from lesional site.

2.3.2 Group C: Normal healthy tissue surgically removed from 6 cleft-lip patients. 


\section{Tissue specimens are divided into 2 parts:}

3.1 Part 1: for Histopathological grading

3.2 Part 2: for Microbial investigation (bacterial culture)

3.1 PART 1: Procedure for histopathological grading:

All the 30 cases were confirmed histopathologically as OSCC.

3.2 PART 2: Half of the sample was used for microbiological culture

\section{Bacterial Culture:}

To determine the number of cells in a bacterial culture, serial dilution was carried out. Bacterial cell numbers need to be reduced, which was done by repeatedly diluting the amount of bacteria in the sample. Total no. of 18 test tubes was taken for serial dilution and each test tube contained $9 \mathrm{ml}$ of distilled water (diluent solution). 6 test tubes each for 3 levels of the cancerous lesion (upper level, middle level \& lower level of the cancerous tissue). A small amount (2 gm) of tissue samples were aseptically macerated with mortar \& pestle and vortexed for 30sec in PBS and the neat suspension was mixed with a diluent solution (distilled water) of the first test tube, and then successive dilutions are made. Then $1 \mathrm{ml}$ each of the last 3 diluted sample test tube (i.e., $10^{-4}$, $10^{-5} \& 10^{-6}$ ) was then spread onto 3 Petri plates i.e., total 9 Petri plates were prepared - 3 each for upper level, middle level \& lower level of lesional tissue respectively.

Onto the petri plates, Luria Bertani (LB) agar was poured by Pour-plate technique. The pour plate technique can be used to determine the number of microbes $/ \mathrm{ml}$ or microbes/gram in a specimen. It has the advantage of not requiring previously prepared plates, and is often used to assay bacterial contamination of foodstuffs. All the agar plates were placed in the incubator at $37^{\circ} \mathrm{c}$ for $24-48 \mathrm{hrs}$. The numbers of bacteria colonies that grew on each plate were counted by colony counter. By working backwards using multiplication with the "dilution factor" (the number of times that you have diluted the bacteria sample with the diluent solution), the numbers of bacteria of the original sample can be determined.

To compute the estimated number of bacteria, the following formula was used:

$$
\text { (1) } B=N / D
$$

$\mathrm{B}=$ number of bacteria

$\mathrm{N}=$ number of colonies counted on a plate

$\mathrm{D}=$ dilution factor (either 1,10 or 100 )

\section{Results:}

The isolation of bacteria from the tissue of the 36 patients was conducted by Traditional Method of Pure Culture with serial dilution. The statistical data was expressed as mean \pm SD. Statistical significance was analyzed using student's unpaired t-test. A p value of less than 0.05 was used to establish statistical significance. A correlation analysis was undertaken using Tukey multiple comparisons test.

\section{Observation \& Discussion}

There have been some studies on the presence of microorganisms on the surface of OSCC lesional tissue as by K. N. Nagy et $\mathrm{al}^{3}$ but in our study we have focused on the presence of viable bacteria within the lesional OSCC tissue as the surface of OSCC tissue is always contaminated due to salivary or instrument contamination during surgery.

In the present study, we have taken tissue to isolate bacteria from 30 OSCC patients at 3 levels- upper, middle \& lower level and considered \& compared with 2 controls- OSCC control \& normal control. Taking into account that the tumor microenvironment may vary according to the depth of the tissue from the surface, we evaluated the bacterial count at 3 levels of the tissue. The present study is unique as it has quantified bacterial count at 3 different levels (upper, middle \& lower levels of cancerous tissue) and a search through standard electronic databases has not been revealed in any such study published till date.

Important mechanisms by which bacterial agents may induce carcinogenesis include chronic infection, immune evasion and immune suppression. It has been seen that bacteria can cause chronic infections or produce toxins that disturb the cell cycle resulting in altered cell growth. The resulting damage to DNA is similar to that caused by carcinogenic agents as the genes that are altered control normal cell division and apoptosis. These processes that encourage the loss of cellular control may be tumor initiators (directly causing mutations) or promoters (facilitating mutations). Tumorigenesis is initiated when cells are released from growth restraints, later promotion results when the immune system is evaded favoring further mutations and increased loss of cell control. ${ }^{1}$

From the OSCC patients, apparently normal tissue was removed at least $5 \mathrm{~cm}$ away from the lesional site where possible. Out of 30 OSCC patients only in 10 patients we were able to remove apparently normal 
tissue at least $5 \mathrm{~cm}$ away from lesional site. These 10 patients were considered as OSCC control. The commonly used controls are usually the clinically apparently non-affected adjacent tissues or sometimes if possible, the anatomical counterpart such as normal appearing buccal mucosa or retro molar pad. These are the best possible baselines for most anatomically discrete pathologies ${ }^{4}$.So we have considered it as our $2^{\text {nd }}$ control (OSCC control). The aerobic bacterial count of OSCC control was higher than the normal control. This may be due to change in oral environment for oral tobacco habit. All the patients had a history of tobacco for a long period of time.

The immune system is an important line of defense for tumor formation of malignancies that express unique antigens. Certain bacterial infections may evade the immune system or stimulate immune responses that contribute to carcinogenic changes through the stimulatory and mutagenic effect of cytokines released by inflammatory cells. These include reactive oxygen species (ROS), interleukin-8 (IL-8), cyclooxygenase-2 $(\mathrm{COX}-2)$ and nitric oxide (NO). Chronic stimulation of these substances along with environmental factors such as smoking, or a susceptible host appears to contribute significantly to carcinogenesis. ${ }^{1}$

We cultivated viable aerobic bacteria from inside of oral squamous cell carcinoma (OSCC) tissue. Immersion in betadine was required to eliminate any viable bacteria on the surface that may have been present due to salivary or instrument contamination during surgery. It has been well established that approximately $50 \%$ of oral bacteria are not cultivable by standard culture technique as used by this study therefore the absolute count of bacteria may not be totally correct. Nevertheless cultivation of bacteria remains a useful diagnostic tool for the detection and identification of viable organisms. ${ }^{5}$ As the surface decontamination indicate that the bacteria isolated from the sample were not carried over from other source such as saliva etc. but were present in the tissue itself.

The cancerous and the apparently normal tissue which was surgically removed from 30 OSCC patients was taken from 3 levels- upper, middle \& lower levels of the lesional site. This was done to quantify and compare the presence of bacteria at different levels of the cancerous tissue. It was assumed that the tumor environment is not uniform throughout the tumor so we expected a varying aerobic bacterial count in the upper, middle \& lower level of tumor. But according to findings in our study and statistical analysis, there was no difference between the aerobic bacterial count of upper, middle and lower level of tumor indicating that aerobic bacterial count in a tumor is not related to any level of the tumor. The present study is unique as it has quantified bacterial count at 3 different levels (upper, middle \& lower levels of cancerous tissue) and a search through standard electronic databases has not been revealed in any such study published till date.

Comparison between the aerobic bacterial count of OSCC tissue at upper, middle and lower level with apparently normal tissue atleast $5 \mathrm{~cm}$ away from the lesional site showed a higher bacterial count in OSCC tissue. This is in accordance with the study carried out by different authors. But only in 2 studies, carried by S J Hooper et al ${ }^{5} \& \mathrm{~K}$. N. Nagy et al ${ }^{3}$, we have found that microbiological method has been used for bacterial estimation.

When comparing OSCC tissue with 2 controls - OSCC control \& normal controls, we found a progressive increase in bacterial count from normal control to OSCC control to OSCC tissue. These changes may be due to changes based on the concept of regional "Field cancerization".

The reasons for the quantitative differences in colonization patterns of microorganisms at different host locations are only partially understood these reasons include difference in nutrient availability, competition among species for binding sites, inter species antagonisms or co-operations and the difference in receptors present on different tissue that permit binding by specific adhesions possessed by different species. Other factors that may party explain the unfavorable microbial shift observed in oral carcinoma are a compromised host response or the irregularity of the lesion surface providing stagnant habits. ${ }^{1}$

\section{Summary \& Conclusion:}

The present study was an attempt to culture aerobic bacteria in OSCC tissue. It was found that viable bacteria were present deep in the OSCC tissue and the quantity present was not dependent on the level of tumor tissue. The Histopathological grade of tumor (WHO), Bryne`s grading and degree of inflammation of the tumor had no correlation with the aerobic bacterial count of the tumor tissue.

The aerobic bacterial count of OSCC tissue was higher than the 2 controls. It was also found that between the 2 controls- the apparently normal tissue atleast $5 \mathrm{~cm}$ away from lesion had a higher bacterial count than the normal healthy non-inflamed tissue. The significant increase in bacterial count is attributed to distinctive change in tumor environment. The cause and effect relationship of the presence of viable bacteria in OSCC tissue could not be ascertained. The colonization of bacteria may be due to oral habits. The fact that the increase in colonization of bacteria may be due to cancer or bacteria are responsible for the cancer is a matter of debate.

It would be useful if future studies include identification of bacteria \& an assessment of microbial load to determine the numerical predominance of selected species. As evidence suggests that bacteria are involved in 
the development of many different cancer increases, it is interesting to speculate that the species isolated from the tumor tissue may play role in the carcinogenesis process, a concept worthy of further investigation.

\section{Acknowledgements:}

We haven't received any grant or other financial support.

\section{References:}

[1] DL Mager. Bacteria and cancer: cause, coincidence or cure: a review. Journal of translational medicine 2006; 4:14.

[2] J Parsonnet. Environ Health Perspect. Bacterial infection as a cause of cancer.1995 Nov; 103(Suppl 8): 263-268.

[3] Nagy KN, Sonkodi I, Szöke I, Nagy E, Newman HN. The microflora associated with human oral carcinomas. Oral Oncol. 1998 Jul; 34(4):304-8.

[4] Rajendran and Sivapathasundharam. Shafer's Text book of oral pathology. Elsevier 2006, 5 th ed. Appendix 1, pg 1233.

[5] Samuel J. Hooper, St John Crean, Michael A. O. Lewis, David A. Spratt, William G. Wade, and Melanie J. Wilson. Viable Bacteria Present within Oral Squamous Cell Carcinoma Tissue. Journal of clinical microbiology. 2006 May; 44(5):1719-1725. 